\title{
ANÁLISIS DE TENDENCIAS CLIMÁTICAS BASADO EN METADATOS DE ESTACIONES DE LA RED DE MEDICIÓN DE LA OFICINA NACIONAL DE METEOROLOGÍA -ONAMET- DE LA REPÚBLICA DOMINICANA
}

Analysis of climate trends based on metadata network stations measuring National Meteorology Office -ONAMET- of the Dominican Republic

\section{Michela Izzo* María Ozoria Zarzuela**}

Resumen: La recolección y el estudio de metadatos constituyen un aspecto fundamental del análisis de series históricas de variables meteorológicas. Los resultados obtenidos para la estación de levantamiento de Santo Domingo, perteneciente a la red de medición de la Oficina Nacional de Meteorología (ONAMET), evidencian que es necesario mejorar la recolección y organización de metadatos asociados a las diferentes estaciones. Las informaciones y datos identificados resultan coherentes con el análisis estadístico-numérico realizado, permitiendo estimar para las series termopluviométricas de Santo Domingo las siguientes tendencias: $6.8 \pm 3.5 \mathrm{~mm} /$ año de los acumulados de precipitación anual en el

\footnotetext{
Es directora ejecutiva de Guakia Ambiente, Santo Domingo. e-mail: michela.izzo@gmail.com

** Experta en retos de la hidrometeorología ante las posibles variaciones del clima y los eventos extremos. E-mail: onamet_zm@yahoo.com
} 
período $1939-2007 ;+3.0 \pm 0.5^{\circ} \mathrm{C}$ y $+1.8 \pm 0.4^{\circ} \mathrm{C}$ respectivamente de la temperatura mínima y máxima promedio anuales en el período 1936-2007.

Palabras claves: Metadatos, series termopluviometricas, variable meteorológica, tendencias climáticas, metadatos, meteorología, ONAMET, República Dominicana.

Abstract: The metadata collection and analysis is a fundamental aspect in the study of historical series of meteo-climatic variables. The results obtained for the Santo Domingo measurement station, which belongs to the National Meteorological Office (ONAMET, according to the Spanish acronym), show that to improve the collection and organization of metadata associated to meteorological stations is a key issue. The identified information and data do not show incoherence with the statistical-numerical analysis which was carried out. The following trends can be estimated for Santo Domingo: $6.8 \pm 3.5 \mathrm{~mm} /$ year for total annual precipitation in the period $1939-2007 ;+3.0 \pm 0.5^{\circ} \mathrm{C}$ and $+1.8 \pm 0.4^{\circ} \mathrm{C}$ respectively for minimum and maximum average annual temperature in the period 1936-2007.

Keywords: Metadata, thermopluviometric series, meteorological variable, climatic trends metadata Meteorology ONAMET, Dominican Republic.

\section{Antecedentes}

En los últimos años se ha verificado una fuerte convergencia respecto a la influencia que las actividades humanas están teniendo sobre el clima (Kelly \& Wigley, 1992; Mann et al., 1998; Crowley \& Lowery, 2000; Barnola et al., 2003; Mann \& Jones, 2003; IPCC, 
2007; Rosenzweig et al., 2008; The Royal Society, 2010) y a nivel científico ha adquirido cada vez más crédito el concepto de "cambio climático", entendido como "cambio del clima atribuido de manera directa o indirecta a la actividad humana que altera la composición de la atmósfera mundial, sumándose a la variabilidad natural del clima observada durante períodos de tiempo comparables" (United Nations, 1992).

Sin tomar en cuenta las causas que están en la base del fenómeno, lo que no se pone en discusión es que en los últimos 150 años, el clima ha estado cambiando, con modificaciones aceleradas (IPCC, 2007), según muestran investigaciones conducidas, en diferentes partes del mundo y a diferente escala, tanto mediante el análisis de series históricas de parámetros meteorológicos (Barnett et al., 1999; Buffoni et al., 1999; Brunetti et al., 2000; Barriendos et al., 2002; Brohan et al., 2006), como mediante investigaciones indirectas de tipo fenológicos, dendrocronológico, palinológico, glaciológico, etc. (Bradley et al., 1999; Labeyrie et al., 2007). A escala global, diferentes estudios han evidenciado un incremento de la temperatura de la superficie terrestre entre $0.3 \mathrm{y}$ $0.6{ }^{\circ} \mathrm{C}$ a partir de la mitad del siglo veinte, con diferencias según la latitud y el hemisferio (Jones et al., 1986a; 1986b; 1986c; Hansen \& Lebedeff, 1987; 1988; Jones, 1988; Parker et al., 1994; Jones \& Briffa, 1992; 1996).

A pesar de que los estudios llevados a cabo hasta ahora han evidenciado fuertes diferencias geográficas y alta complejidad del fenómeno, los resultados obtenidos muestran que la tendencia al calentamiento es generalizada, con una aceleración del incremento en el transcurso de los años 90 del siglo veinte, cuya década resultó ser la más cálida del último milenio (IPCC, 2007). Mientras que se observa un general incremento de las temperaturas (IPCC, 2007), las precipitaciones muestran comportamientos que dependen de la región y las características geográficas y topográficas del sitio analizado (Zveryaev \& Chu, 2003; IPCC, 2007). 
En República Dominicana, las previsiones basadas en modelos numéricos han identificado escenarios que prevén un crecimiento generalizado de las temperaturas y una reducción significativa de las precipitaciones (SEMARENA, 2004; 2009; Parish et al., 2008). Estudios recientes (Izzo, 2011), basados en análisis de series históricas registradas por la red de medición disponible en el país, han confirmado el incremento de la temperatura en todo el territorio dominicano, mientras que para las precipitaciones han evidenciado un comportamiento dependiente de la región climática considerada. Dichos resultados, obtenidos realizando un análisis de calidad de las series y sucesiva homogeneización de tipo exclusivamente numérico (Izzo, 2011), debido a la falta de informaciones sobre el historial de las estaciones consideradas, necesitan ser verificados y eventualmente ajustados, una vez que dichos datos estén disponibles.

\section{Problema de la investigación}

En el ámbito climatológico, la disponibilidad de una base de datos meteorológicos amplia es una condición necesaria, pero no suficiente para una reconstrucción eficaz de las evoluciones del clima en un área determinada. Para fines de identificar las tendencias climáticas eventualmente presentes en una serie de medidas es indispensable poder aislar la señal climática de cambios ligados al ambiente de medición (Easterling \& Peterson, 1995; Alexadersson, 2001; Bhöm et al., 2001; Auer et al., 2005; Maugeri et al., 2006). En este sentido, es imprescindible poder contar con la disponibilidad de metadatos, o sea, con informaciones detalladas sobre la red de medición, las cuales incluyan:

- Creación, organización y desarrollo de la red de levantamiento;

- Evolución de las metodologías y herramientas de medición adoptadas en cada estación; 
- Historia detallada de cada estación de medición, incluyendo informaciones sobre el entorno espacial del punto de levantamiento.

La homogeneización de datos climatológicos consiste en su corrección de manera que sean hechos confrontables en todo el período de medición, o sea hacer como si hubiesen sido siempre observados bajo las condiciones operativas existentes en el período de referencia. Los métodos de homogeneización se clasifican en directos o basados en la reconstrucción de los metadatos asociados a la serie medida, e indirectos, o sea, basados en la aplicación de técnicas estadísticas apropiadas, que permitan identificar puntos de discontinuidad significativos que estén presentes en la serie analizada, normalmente mediante una comparación con otras series de mediciones (Alexandersson, 2001; Nanni, 2002; Camuffo et al., 2006; Maugeri et al., 2006). El procedimiento ideal consiste en la aplicación conjunta de dichos enfoques, de manera que el análisis estadístico sea complementado, respaldado y verificado en base a las informaciones específicas que estén disponibles sobre los cambios ocurridos y documentados durante el funcionamiento de la estación (Brunetti et al., 2002; Nanni, 2002; Maugeri et al., 2006; Domonkos \& Stepánek, 2009).

El tema de la homogeneización de las series climatológicas, así como aquel relativo a las más apropiadas técnicas a usarse para obtener datos homogéneos es objeto de debate en el seno de la comunidad científica internacional, no contándose al momento con una metodología que pueda considerarse válida en absoluto. Por dicha razón, hay varios estudios que confrontan diferentes enfoques y metodologías (Nanni, 2002; Wijngaard et al., 2003; Maugeri et al., 2006; Reeves et al. 2007; Beaulieu et al., 2009; Costa \& Soares, 2009).

En República Dominicana actualmente la Oficina Nacional de Meteorología (ONAMET) tiene en desarrollo un programa orientado a la recolección y organización de metadatos relativos a las estaciones de la red de medición nacional, cuya base de datos está 
en fase de organización, así como la recolección de las informaciones. $\mathrm{Al}$ presente, no se cuenta con historiales detallados de cada estación de la red de medición meteo-climática nacional. Debido a esta limitación, la homogeneización que ha precedido todos los análisis climáticos realizados hasta el momento, han sido basado exclusivamente en procedimientos indirectos (Izzo, 2011).

En este contexto, para fines de mejorar el conocimiento climatológico a nivel de país, verificando y profundizando los resultados obtenidos hasta ahora es indispensable el trabajo de recolección y sistematización de los metadatos relativos a cada una de las estaciones de la red nacional de levantamiento meteo-climático.

El análisis, que en el futuro se prevé extender a otras estaciones de la red de medición de la ONAMET, en el presente estudio está enfocado en la estación de Santo Domingo. Las razones de dicha elección residen en los siguientes puntos: Santo Domingo, como capital y principal centro económico, político e industrial del país, tiene una importancia estratégica en términos de definición de las tendencias climáticas históricas, para fines de definir medidas de adaptación a futuros cambios; la serie termopluviométrica de Santo Domingo es una de las de mejor calidad, según lo revelado por el análisis estadístico-numérico realizado en los estudios anteriores (Izzo, 2011). En este sentido, la estación de Santo Domingo presenta las características apropiadas para poder sentar las bases para un análisis climatológico fundamentado en metadatos adecuadamente recolectados y organizados.

\section{Materiales y métodos}

La investigación ha sido estructurada en dos etapas principales: La primera parte del estudio ha sido caracterizada por la recolección de datos históricos sobre el funcionamiento de la estación de Santo Domingo y de otras informaciones relevantes para fines de análisis climatológicos. Esta fase ha sido desarrollada en estrecha colaboración con el personal técnico de la ONAMET y demás 
actores clave en el proceso de recolección de datos y metadatos meteo-climáticos, incluyendo entrevistas con operadores y personas informadas sobre el funcionamiento de las estaciones. De la misma manera, se ha procedido a la recuperación de los registros de la estación y sucesivo análisis, para fines de identificar los cambios que hayan ocurrido en la misma.

La ONAMET está desarrollando un programa orientado a la recolección y organización de metadatos relativos a las estaciones de la red de medición nacional. Por esta razón, los técnicos y encargados de departamento presentes sugirieron un cambio del título de la investigación, de manera que pudiera destacarse que el estudio se inserta en un proceso ya existente. Para tales fines, se propone el siguiente título: "Análisis de tendencias climáticas basado en metadatos de estaciones de la red de medición de la Oficina Nacional de Meteorología (ONAMET) de la República Dominicana".

En la segunda fase del estudio, los datos y metadatos recolectados y sistematizados, han sido analizados con tal de revisar los resultados de la homogeneización basada en métodos numéricos, verificando las tendencias históricas identificadas en estudios anteriores (Izzo, 2011).

Específicamente, se usaron las técnicas del Penalized Maximal t-Test (PMT) y el Penalized Maximal F-Test (Wang, 2003; Wang et al., 2007; Wang, 2008a; 2008b; Wang et al., 2010) para la identificación de los puntos de discontinuidad presentes y el Quantile Matching Adjustement (Wang, 2009; Wang et al., 2010) para la homogeneización de las series analizadas. La identificación de los puntos de discontinuidad está basada en la identificación de cambios en la media de las series de datos, tomando en cuenta la posición relativa de cada posible discontinuidad. De esta manera, se reduce el efecto de las dimensiones desiguales de la muestra sobre la potencia de la identificación (Wang et al., 2007). La homogeneización se lleva a cabo mediante un ajuste de la serie de base a las distribuciones 
empíricas de todos los segmentos de la misma serie sin tendencias, con relación a la frecuencia empírica de los datos a corregir (Wang, 2009; Wang et al., 2010). Los análisis estadísticos para la homogeneización de las series han sido conducidos mediante el uso del software R (R Development Core Team, 2010).

La homogeneización realizada mediante la aplicación de métodos indirectos está basada esencialmente en la construcción de una serie de referencia, con la cual pueden compararse las estaciones analizadas (Maugeri et al., 2006). Dicha serie se construye como promedio de los datos de las estaciones más similares, ubicadas en áreas con características climáticas análogas. El número de las estaciones usadas para construir la serie de referencia debe ser suficientemente grande para mascarar posibles discontinuidades de los datos de referencia, dependiendo de todas maneras de factores prácticos, como son la densidad de la red de medición y la cantidad y calidad de datos disponibles.

Para la identificación de grupos de estaciones sobre las cuales construir las series de referencia, en concreto, ha sido aplicado un clustering jerárquico aglomerativo, basado en la métrica de la distancia euclidea y un método de aglomeración de tipo completo (Anderberg, 1973; Hastie et al., 2009). La serie de referencia ha sido obtenida mediante los datos de las series que pertenecen al mismo clúster.

La investigación ha sido conducida sobre las series mensuales de precipitación y temperatura mínima y máxima, extendiéndose desde finales de los años treinta del siglo pasado hasta el 2007. Los grupos de estaciones resultantes del clustering jerárquico han sido luego usados para completar las series, operando mediante la técnica de la Multiple Imputation (Rubin, 1976; 1987), aplicada mediante el uso del paquete Amelia II del software $\mathrm{R}$ (Honaker et al., 2009), el cual la implementa mediante procedimientos de bootstrapping (Davison \& Hinkley, 1997). 
La calidad del dato termométrico ha tenido reflejos en la imposibilidad de procesar los datos según la metodología definida anteriormente y aplicada a las series pluviométricas. En particular, debido al número limitado de estaciones disponibles y de la reducida calidad del dato, para fines de establecer su homogeneidad y proceder eventualmente a su homogeneización, las series de temperatura mínima y máxima han sido analizadas individualmente, sin el uso de series de referencia.

Los resultados de la recolección y análisis de evidencias históricas relativa a la estación de medición de Santo Domingo han sido usados para evaluar la coherencia de los resultados obtenidos con los métodos numéricos descritos en este artículo.

Todo el proceso de investigación es realizado en coordinación con la Oficina Nacional de Meteorología (ONAMET) y en colaboración con el Programa de Pequeños Subsidios (PPS-SGP) del Fondo para el Medio Ambiente Mundial (FMAM), cuya experiencia demostrada en el tema de cambio climático, análisis territoriales y procesos comunitarios se convirtió en un aporte valioso en el proceso de reconstrucción previsto. El PPS-SGP proporciona apoyo técnico, además de la oficina y otras facilidades indispensables para llevar a cabo el trabajo de investigación. Se ha contado también con el apoyo del personal docente de informática y estadística de la Universidad Parthenope de Nápoles (Italia).

\section{Resultados}

En la actualidad los datos organizados por la ONAMET recogen especialmente los cambios registrados por los encargados de la estación de Santo Domingo, así como las fechas de la entrada en funcionamiento de los principales instrumentos de medición. Se dispone de información limitada sobre los cambios ocurridos en la estación en términos de ubicación y sustitución o mantenimiento de los instrumentos de medición. Un evento sucedido en agosto de 2000 generó la destrucción de una parte de la información 
relacionada con documentos archivados, pero al parecer, los archivos relativos a la estación de Santo Domingo fueron salvados.

En una de las entrevistas llevadas a cabo, se tuvo la noticia de un estudio, titulado "Historia de la meteorología", elaborado por Vidal et al. que recogería informaciones importantes sobre los cambios ocurridos en la instrumentación de las estaciones ONAMET. Sin embargo, las personas y las investigaciones llevadas a cabo hasta el momento todavía no han permitido conseguir una copia de dicho estudio ni conocer el año exacto de la publicación.

Las dificultades tenidas en el proceso de reconstrucción de los cambios principales ocurridos en el transcurso de la historia de la estación de levantamiento revelan que es necesario mejorar el proceso de documentación de las modificaciones que ocurren en los instrumentos y en el lugar de medición, así como de su entorno. En este sentido, se considera muy atinado y valioso que la ONAMET cuente con un departamento de metadatos, el cual podrá evitar la pérdida de informaciones fundamentales para futuros análisis climatológicos.

El clustering jerárquico evidencia que la serie pluviométrica de Santo Domingo presenta características similares a otras cuatro series, las cuales incluyen las de Barahona, San Pedro de Macorís, Rancho Arriba y San Cristóbal (Izzo, 2011).

El análisis de las series del grupo de Santo Domingo, conducida en base a la referencia de la serie promedio homogeneizada ha evidenciado un punto de discontinuidad en mayo de 1963.

En el caso de las series termométricas, el análisis de homogeneidad llevado a cabo revela un punto de discontinuidad en 1949 para la temperatura mínima, mientras que no evidencia discontinuidad en el caso de las temperaturas máximas. (Izzo, 2011).

Los resultados de las investigaciones que se realizaron para recolectar metadatos concernientes a la serie termopluviométrica de Santo 
Domingo quedan sintetizados en el cuadro 1 , donde se presenta el cronograma de los eventos relativos a la estación de medición del cual se tuvo noticia durante el estudio.

La escasez de fuentes de informaciones relativas a la historia de la estación y de los acontecimientos con potencial efecto sobre las variables meteorológicas medidas hace que al estado actual no se pueda considerar determinante ninguno de los eventos identificados. Se considera relevante el punto de discontinuidad identificado en 1949 en la serie de temperatura mínima. Dicho punto de discontinuidad podría estar asociado al traslado de la estación de la Zona Colonial a Punta Torrecilla, a la cercanía hidrográfica del Río Ozama. El cambio de exposición y las condiciones más rurales podrían haber tenido una influencia, especialmente sobre el registro de las temperaturas mínimas.

En base a las evidencias recolectadas no se evidencian incoherencias respecto al análisis estadístico-numérico llevado a cabo, lo que por el momento permite aceptar las tendencias estimadas para las variables de temperatura y precipitaciones: $6.8 \pm 3.5 \mathrm{~mm} /$ año de los acumulados de precipitación anual en el período 1939-2007; $+3.0 \pm 0.5{ }^{\circ} \mathrm{C}$ y $+1.8 \pm 0.4{ }^{\circ} \mathrm{C}$ respectivamente de la temperatura mínima y máxima promedio anuales en el período 1936-2007. Los resultados obtenidos en esta investigación destacan la importancia de seguir profundizando aspectos relativos a la historia del levantamiento de datos meteoclimáticos en la República Dominicana.

El análisis de los cambios ocurridos en la extensión del núcleo urbano de la ciudad de Santo Domingo revela una ampliación significativa del área urbana. En el período 1968-2003, la superficie urbana de Santo Domingo tuvo un crecimiento promedio anual del $25 \%$, pasando de $28 \mathrm{~km}^{2}$ en al 1968 a $124 \mathrm{~km}^{2}$ en el 1983 , hasta $287 \mathrm{~km}^{2}$ en el 2003 (Figura 1). 


\section{Cuadro 1}

\section{Cronograma de los principales eventos de la estación ONAMET de Santo Domingo.}

\begin{tabular}{|c|c|c|}
\hline Fecha & Evento & Observaciones \\
\hline 1909 & $\begin{array}{l}\text { Primeros informes de valores de } \\
\text { precipitación. }\end{array}$ & $\begin{array}{l}\text { Las observaciones se realizaban en dife- } \\
\text { rentes zonas de la ciudad: Calle Colón, } \\
\text { Calle Padre Billini, Calle José Gabriel García, } \\
\text { Avenida George Washington. }\end{array}$ \\
\hline 1915 & $\begin{array}{l}\text { Nombramiento nuevo director Servicio } \\
\text { Meteorológico Nacional: Francisco } \\
\text { Contrain. }\end{array}$ & \\
\hline 1917 & $\begin{array}{l}\text { Primeros informes de valores de } \\
\text { temperaturas y presión. }\end{array}$ & $\begin{array}{l}\text { Las observaciones se realizaban en dife- } \\
\text { rentes zonas de la ciudad: Calle Colón, } \\
\text { Calle Padre Billini, Calle José Gabriel García, } \\
\text { Avenida George Washington. }\end{array}$ \\
\hline 1920 & $\begin{array}{l}\text { Nombramiento nuevo director Servicio } \\
\text { Meteorológico Nacional: Ramón Jansen. }\end{array}$ & \\
\hline 1924 & $\begin{array}{l}\text { Nombramiento nuevo director Servicio } \\
\text { Meteorológico Nacional: Aurelio Ortorí. }\end{array}$ & \\
\hline 1930 & $\begin{array}{l}\text { Encargado estación: Juan Bautista } \\
\text { Cambiaso Váldez (inicio período). }\end{array}$ & \\
\hline 1931 & Inicio mediciones pluviométricas. & \\
\hline 1931 & Inicio observaciones de nubosidad. & \\
\hline 1935 & $\begin{array}{l}\text { Terminación período del director } \\
\text { Aurelio Ortorí. }\end{array}$ & \\
\hline 1936 & $\begin{array}{l}\text { Conversión en estación } \\
\text { termopluviométrica. }\end{array}$ & \\
\hline 1940 & $\begin{array}{l}\text { Encargado estación: Juan Bautista } \\
\text { Cambiaso Váldez (terminación } \\
\text { período). }\end{array}$ & \\
\hline 1945 & $\begin{array}{l}\text { Nombramiento nuevo director Servicio } \\
\text { Meteorológico Nacional: Sebastián } \\
\text { Robiou Valverde. }\end{array}$ & \\
\hline 1947 & $\begin{array}{l}\text { Inicio observaciones de dirección del } \\
\text { viento. }\end{array}$ & \\
\hline 1948 & $\begin{array}{l}\text { Traslado de la estación en la parte } \\
\text { oriental del Río Ozama: Punta Torre- } \\
\text { cilla, posiblemente en los terrenos de } \\
\text { la Marina de Guerra. }\end{array}$ & $\begin{array}{l}\text { Sucesivamente, la estación fue movida a } \\
\text { los terrenos de la actual ONAMET. La } \\
\text { estación estaba ubicada en la parte delan- } \\
\text { tera del recinto de la ONAMET. }\end{array}$ \\
\hline 1948 & $\begin{array}{l}\text { Nombramiento nuevo director Servicio } \\
\text { Meteorológico Nacional: Juan Moliné. }\end{array}$ & \\
\hline 1951 & $\begin{array}{l}\text { Encargado estación: Víctor Manuel } \\
\text { Matos Pérez (inicio período). }\end{array}$ & $\begin{array}{l}\text { Observadores en el mismo período (1951- } \\
\text { 1956): César del Villar, Rafael Pérez } \\
\text { Diloné, Guarocuya Moliné, Andrés Cam- } \\
\text { pusano Romero. }\end{array}$ \\
\hline 1951 & $\begin{array}{l}\text { Inicio mediciones de velocidad del } \\
\text { viento, velocidad máxima del viento, } \\
\text { humedad relativa. }\end{array}$ & \\
\hline
\end{tabular}


Análisis de tendencias climáticas basado en metadatos de estaciones de la red de medición de la Oficina Nacional de Meteorología de la República Dominicana

\begin{tabular}{|c|c|c|}
\hline Fecha & Evento & Observaciones \\
\hline 1956 & $\begin{array}{l}\text { Encargado estación: Víctor Manuel } \\
\text { Matos Pérez (terminación período). }\end{array}$ & \\
\hline 1956 & $\begin{array}{l}\text { Traslado de la estación de los terrenos } \\
\text { de la Marina de Guerra al recinto de } \\
\text { la ONAMET (cerca de la mata de } \\
\text { mango). }\end{array}$ & $\begin{array}{l}\text { Podría haber problemas con los datos de } \\
\text { temperatura registrados en ese período, } \\
\text { debido a la quema de basura que en hora de } \\
\text { la mañana se realizaba constantemente. } \\
\text { Hasta los años '90 ocasionaron también } \\
\text { problemas los árboles que rodeaban la garita. }\end{array}$ \\
\hline 1956 & $\begin{array}{l}\text { La estación pasa a un grupo de técni- } \\
\text { cos que realizan mediciones durante } \\
\text { las } 24 \text { horas del día, a intervalo de } \\
\text { tres horas. }\end{array}$ & $\begin{array}{l}\text { Encargado de la estación: } \\
\text { César del Villar. }\end{array}$ \\
\hline 1956 & $\begin{array}{l}\text { Nombramiento nuevo director Servicio } \\
\text { Meteorológico Nacional: Juan Batista } \\
\text { Cambiaso Valdez. }\end{array}$ & \\
\hline $\begin{array}{l}\text { Mayo } \\
1958\end{array}$ & $\begin{array}{l}\text { Nombramiento de nuevo encargado: } \\
\text { Julio César Román Carbucia. }\end{array}$ & $\begin{array}{l}\text { Técnicos: Andrés Campusano Romero, } \\
\text { William Desueza F., Juan A. Sewer, Aníbal } \\
\text { Germán Martínez, Richard Veloz Cabral. }\end{array}$ \\
\hline $\begin{array}{l}\text { Octubre } \\
1959\end{array}$ & $\begin{array}{l}\text { Terminación período del encargado } \\
\text { Julio César Román Carbucia. }\end{array}$ & \\
\hline $\begin{array}{l}\text { Noviembre } \\
1959\end{array}$ & $\begin{array}{l}\text { Nombramiento de nuevo encargado: } \\
\text { William Desueza. }\end{array}$ & $\begin{array}{l}\text { Técnicos: Juan A. Sewer, Aníbal Germán } \\
\text { Martínez, Richard Veloz Cabral. }\end{array}$ \\
\hline $\begin{array}{l}\text { Primera } \\
\text { mitad de } \\
\text { los ' } 60\end{array}$ & $\begin{array}{l}\text { Traslado de la estación del lado } \\
\text { oriental en la parte frontal del edificio } \\
\text { ONAMET al lado occidental de la misma. }\end{array}$ & \\
\hline 1960 & Inicio mediciones de evaporación. & Disponibles hasta el 1987. \\
\hline 1961 & $\begin{array}{l}\text { En coincidencia con la muerte de } \\
\text { Trujillo, hubo el abandono de la Ofi- } \\
\text { cina de parte de su personal, el cual } \\
\text { duró alrededor de una semana. }\end{array}$ & \\
\hline 1962 & $\begin{array}{l}\text { Terminación período del director } \\
\text { Juan Batista Cambiaso Valdez. }\end{array}$ & \\
\hline $\begin{array}{c}\text { Enero } \\
1963\end{array}$ & $\begin{array}{l}\text { Nombramiento de nuevo encargado: } \\
\text { Aníbal Germán Martínez. }\end{array}$ & $\begin{array}{l}\text { Técnicos: Richard Veloz Cabral, Carlos } \\
\text { Vinicio Acevedo, Julio Ernesto Concha. }\end{array}$ \\
\hline 1965 & $\begin{array}{l}\text { En coincidencia con la ocupación } \\
\text { estadounidense, hubo un día de inte- } \\
\text { rrupción en el trabajo de la Oficina. }\end{array}$ & \\
\hline 1968 & Inicio mediciones de tensión de vapor. & Disponibles hasta el 1987. \\
\hline $\begin{array}{l}\text { Julio } \\
1974\end{array}$ & $\begin{array}{l}\text { Nombramiento de nuevo encargado: } \\
\text { Sergio Heredia. }\end{array}$ & $\begin{array}{l}\text { Técnicos: Julio Ernesto Concha, Ángel } \\
\text { Mercado Martínez, Carlos Vinicio Acevedo, } \\
\text { Máximo Ega. }\end{array}$ \\
\hline 1975 & Inicio mediciones de presión. & \\
\hline 1981 & $\begin{array}{l}\text { Firma de reportes mensuales por } \\
\text { Ángel Mercado y Sergio Heredia. }\end{array}$ & Hasta 1982 (incluido). \\
\hline 1983 & $\begin{array}{l}\text { Firma de reportes mensuales por } \\
\text { Sergio Heredia y Jullio Ernesto Concha. }\end{array}$ & Hasta 1985 (incluido). \\
\hline 1984 & Inicio mediciones de radiación solar. & Disponibles hasta el 1995. \\
\hline 1986 & $\begin{array}{l}\text { Inicio firma de reportes mensuales } \\
\text { por Máximo Ega. }\end{array}$ & Hasta 1988 (incluido). \\
\hline
\end{tabular}




\begin{tabular}{|c|c|c|}
\hline Fecha & Evento & Observaciones \\
\hline $\begin{array}{l}\text { Inicio de } \\
\text { los ' } 90\end{array}$ & $\begin{array}{l}\text { Huelga en la Oficina Nacional de } \\
\text { Meteorología. }\end{array}$ & \\
\hline $\begin{array}{l}\text { Enero } \\
1990\end{array}$ & $\begin{array}{l}\text { Nombramiento nuevo encargado: } \\
\text { Máximo Ega. }\end{array}$ & $\begin{array}{l}\text { Sergio Heredia dejó su cargo a finales de } \\
1989 .\end{array}$ \\
\hline $\begin{array}{l}\text { Junio } \\
1990\end{array}$ & $\begin{array}{l}\text { Traslado de la estación en la parte } \\
\text { trasera del recinto de la ONAMET. }\end{array}$ & \\
\hline 1990 & $\begin{array}{l}\text { Firma de reportes mensuales por } \\
\text { Máximo Ega y Juana A. Sillé. }\end{array}$ & Hasta 1991 (incluido). \\
\hline 1991 & $\begin{array}{l}\text { Nombramiento de nuevo encargado: } \\
\text { Juana A. Sillé. }\end{array}$ & \\
\hline 1992 & $\begin{array}{l}\text { Firma de reportes mensuales por } \\
\text { Miguelina Guzmán y Juana Sillé. }\end{array}$ & Hasta 1994 (incluido). \\
\hline $\begin{array}{l}\text { Junio } \\
1995\end{array}$ & $\begin{array}{l}\text { Nombramiento de nuevo encargado: } \\
\text { Pedro García Marión. }\end{array}$ & $\begin{array}{l}\text { Se habla sólo de "mediado de año", no se } \\
\text { precisa el mes. }\end{array}$ \\
\hline 1996 & $\begin{array}{l}\text { Firma de reportes mensuales por } \\
\text { Joselín Concepción, Pedro García } \\
\text { Marión, Juana Sillé. } \\
\end{array}$ & \\
\hline $\begin{array}{c}\text { Enero } \\
1999 \\
\end{array}$ & $\begin{array}{l}\text { Nombramiento de nuevo encargado: } \\
\text { Pablo A. Batista. }\end{array}$ & $\begin{array}{l}\text { Técnicos: Elis Aurora Pérez, Juana Sillé, } \\
\text { Pedro García Marión. }\end{array}$ \\
\hline 01/09/2000 & $\begin{array}{l}\text { Nombramiento de nuevo encargado: } \\
\text { Máximo Ega. }\end{array}$ & $\begin{array}{l}\text { Técnicos: Pedro García Marión, Elis A. } \\
\text { Pérez, Rafaela Concepción, María Josefina } \\
\text { Pool, María Altagracia Zabala, Francisco } \\
\text { Holguín, Lucía López, Daniel Peña, Edwin } \\
\text { Pérez, Henry Agramante, Modesta Peña, } \\
\text { Rafael Sánchez Capellán. }\end{array}$ \\
\hline 2011 & $\begin{array}{l}\text { Actual clasificación y ubicación de la } \\
\text { estación }\end{array}$ & $\begin{array}{l}\text { Estación sinóptica convencional. Las coor- } \\
\text { denadas de la estación son las siguientes: } \\
\text { LAT } 18.474277 \text { N LONG } 69.871341 \text { O } \\
\text { ALT } 14 \mathrm{msnm} \text {. }\end{array}$ \\
\hline
\end{tabular}

Fuente: Elaboración propia.

Analizando la posición de la estación de medición ONAMET, se puede observar que hasta el 1948 fue ubicada en diferentes sitios de la zona colonial (Figura 1), en una zona urbana rodeada de ambientes rurales, siendo bordeada por el Mar Caribe en el Sur y áreas rurales en el este. A partir de mediados de los años 60, en una fecha que todavía queda por definir, la estación fue trasladada en la zona de Los Mameyes, en la rivera izquierda del Río Ozama, siendo ubicada inicialmente en las edificaciones de la Marina de Guerra y sucesivamente en el recinto de la ONAMET, donde se siguen llevando a cabo las mediciones. Dicha área, que en el 1968 era prevalentemente rural, fue progresivamente urbanizándose, hasta llegar a sus dimensiones actuales. 
Análisis de tendencias climáticas basado en metadatos de estaciones de la red de medición de la Oficina Nacional de Meteorología de la República Dominicana

Figura 1 - Ampliaciones del área urbana de Santo Domingo y posición de la estación de levantamiento meteorológico ONAMET, en el período 1968-2003

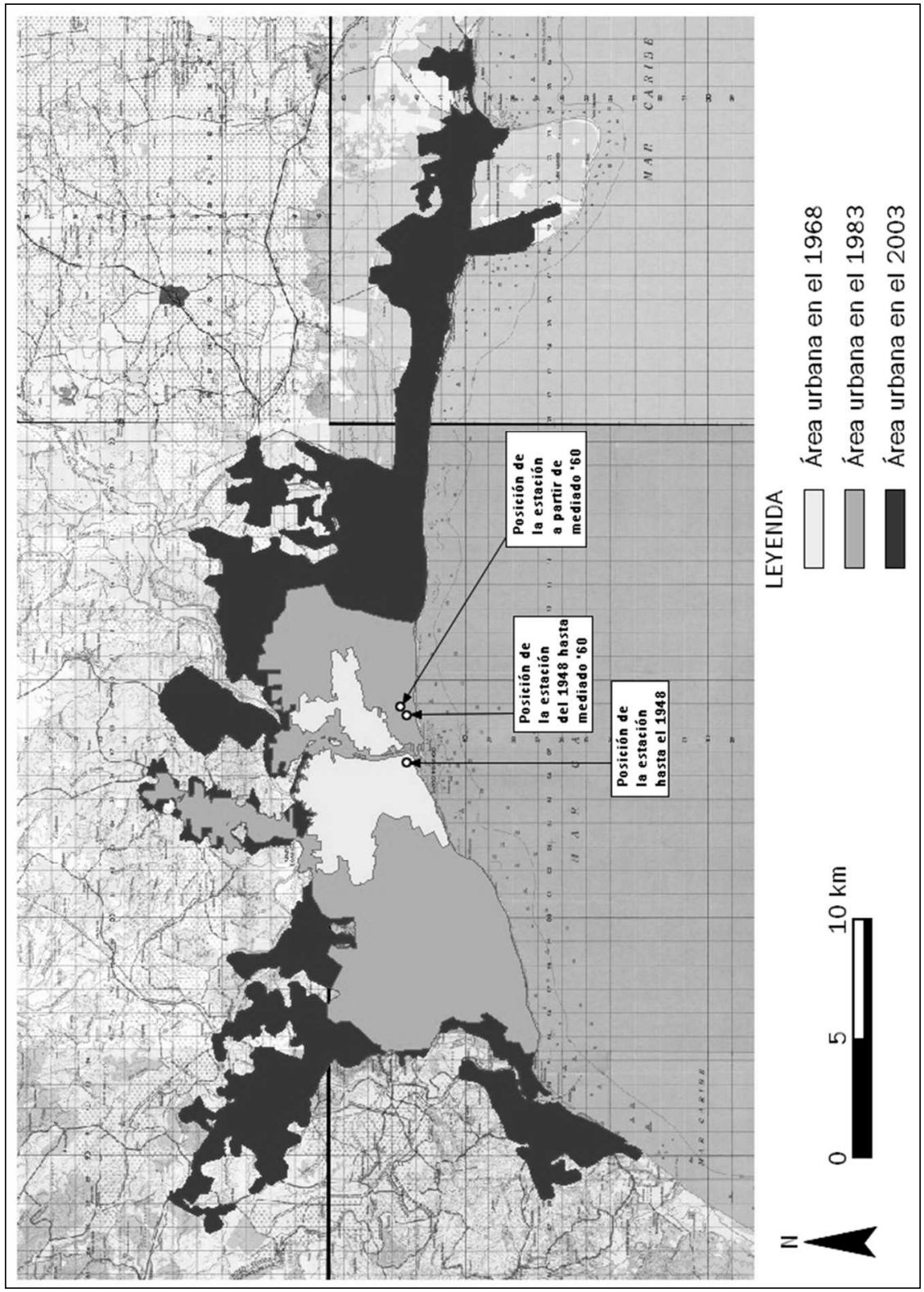

Ciencia y Sociedad 2014; 39(1): 195-219 209 
Cabe destacar que la estación de medición ha estado ubicada siempre en un contexto próximo a la costa: el ámbito territorial de la zona colonial, en términos de exposición y altura, presenta similitudes significativas con aquel de la zona de Los Mameyes, donde, con pocas variaciones, la estación ha permanecido hasta el momento. Dicha área, que ha ido progresivamente urbanizándose, de todas maneras no presenta edificios altos, ni complejos industriales que puedan haber alterado significativamente los parámetros meteorológicos registrados.

\section{Conclusiones}

El presente estudio ha contribuido a mejorar la disponibilidad de metadatos relativos a la estación de Santo Domingo, también ha identificado algunos de los eventos que potencialmente tienen una influencia sobre el levantamiento de variables meteorológicas, como paso fundamental en el proceso de identificación y análisis de las tendencias climáticas estimadas.

La investigación llevada a cabo muestra que es necesario mejorar el proceso de recolección y sistematización de metadatos, puesto que al presente es significativa la carencia de documentación específica, lo cual permite solo en parte evaluar la coherencia de los resultados del análisis estadístico-numérico para la homogeneización de las series termopluviométricas estudiadas.

Una de las líneas de investigación que se considera relevante para el futuro es la cuantificación del efecto de calor urbano en un contexto tropical, especialmente caribeño. Un campo importante es el desarrollo de un modelo a partir de la comparación de datos de temperatura (mínima y máxima) registrado, en un número suficientemente grande de casos, en contexto urbano y no urbano de diferentes ciudades, ubicadas en zonas climáticas similares.

Paso siguiente será la recolección de metadatos de las estaciones (Barahona, San Pedro de Macorís, Rancho Arriba y San Cristóbal) 
que, en base al cluster analysis, han podido presentar series similares a las registradas en Santo Domingo. Extender el análisis llevado a cabo en la presente investigación a las demás estaciones permitirá recolectar evidencias que confirmen la coherencia de la herramienta de agrupación usada en el estudio.

\section{Referencias bibliográficas}

Alexandersson, H. (2001). Homogenisation of climate data, difficult but necessary. En M. Brunet India \& D. López Bonillo Detecting and Modelling Regional Climate Change. (3-12). Berlín: Springer-Verlag.

Anderberg, M. R. (1973). Cluster analysis for applications. New York: Academic Press.

Auer, I., Böhm, R., Jurkovic, A., Orlik, A., Potzmann, R., Schöner, W., Ungersböck, M. et al. (2005). A new instrumental precipitation dataset for the greater Alpine region for the period 1800-2002. International Journal of Climatology, 25, 139-166.

Barnett, T. P., Hasselmann, K., Chelliah, M., Delworth, T., Hegerl, G., Jones, P. D. (1999). Detection and attribution of recent climate change: A status report. Bulletin of the American Meteorological Society, 80(12), 2631-2659.

Barnola, J.-M., Raynaud, D., Lorius, C., Barkov, N. I. (2003). Historical $\mathrm{CO}_{2}$ record from the Vostok ice core. In Trends: A compendium of data on global change. Tennessee: Carbon Dioxide Information Analysis Center, Oak Ridge National Laboratory.

Barriendos, M., Martín-Vide, J., Peña, J. C., Rodríguez, R. (2002). Daily meteorological observations in Cádiz - San Fernando: Analysis of the documentary sources and the instrumental data content (1786-1996). Climatic Change, 53, 151-170. 
Beaulieu, C., Seidou, O., Ouarda, T. B. M., Zhang, X. (2009). Intercomparison of homogenization techniques for precipitation data continued: Comparison of two recent Bayesian change point models. Water Resources Research, 45, 8410-8422.

Bhöm, R., Auer, I., Brunetti, M., Maugeri, M., Nanni, T., Schöner, W. (2001). Regional temperature variability in the European Alps 1760-1998 from homogenized instrumental time series. International Journal of Climatology, 21, 1779-1801.

Bradley, N. L., Leopold, A. C., Ross, J., Huffaker, W. (1999). Phenological changes reflect climate changes in Wisconsin. Proceedings of the National Academy of Sciences of the United States of America, 96(17), 9701-9704.

Brohan, P., Kennedy, J. J., Harris, I., Tett, S. F., Jones, P. D. (2006). Uncertainty estimates in regional and global observed temperature changes: A new dataset from 1850. Journal of Geophisical Reserach, 111, 12106.

Brunetti, M., Maugeri, M., Nanni, T. (2000). Variations of temperature and precipitation in Italy from 1866 to 1995. Theoretical and Applied Climatology, 65, 165-174.

Brunetti, M., Buffoni, L., Fassina, A., Iafrate, L., Lentini, G., Mangianti, F. (2002). Acquisizione, esame critico ed analisi di serie storiche italiane per lo studio delle variazioni del clima. In Progetto CLIMAGRI, Relazione Tecnico-Scientifica ( $2^{\circ}$ anno). Recuperado de http:// climagri.entecra.it/9D2C 5095-06B3-4716-9DC0-B425E8EFAEE9/FinalDownload/ DownloadId-CBF6ADEDAC3F2177465675EC22168C4C/ 9D2C5095-06B3-4716-9DC0-B425E8EFAEE9/relazioni Finali/2anno/Maugeri_relazione2anno.pdf

Buffoni, L., Maugeri, M., Nanni, T. (1999). Precipitation in Italy from 1833 to 1996. Theoretical and Applied Climatology, 63, 33-40. 
Análisis de tendencias climáticas basado en metadatos de estaciones de la red de medición de la Oficina Nacional de Meteorología de la República Dominicana

Camuffo, D., Cocheo, C., Sturaro, G. (2006). Corrections of systematic errors, data homogenisation and climatic analysis of the Padova pressure series (1725-1999). Climate Change, 78, 493-514.

Costa, A. C., \& Soares, A. (2009). Homogenization of climate data: Review and new perspectives using geostatistics. Mathematical Geosciences, 41(3), 291-305.

Convención Marco de las Naciones Unidas sobre Cambio Climático. (2004). Santo Domingo. Secretaría de Estado de Medio Ambiente y Recursos Naturales.

Convención Marco de las Naciones Unidas sobre Cambio ClimáticoProyecto de Cambio Climático de la República Dominicana. (2009). Santo Domingo: Secretaría de Medioambiente y Recursos Naturales y Programa de las Naciones Unidas para el Desarrollo.

Crowley, T. J., \& Lowery, T. (2000). How warm was the Medieval Warm Period? A comment on "man-made" versus natural climate change. Atmospheric Biology, 29, 51-54.

Davison, A. C., \& Hinkley, D. V. (1997). Bootstrap methods and their application. Cambridge: Cambridge University Press.

Development Core Team. (2010). R: A Language and Environment for Statistical Computing. R Foundation for Statistical Computing. Recuperado de http://www.R-project.org/

Domonkos, P., \& Stepánek, P. (2009). Statistical characteristics of detectable inhomogeneities in observed meteorological time series. Studia Geophysica et Geodaetica, 53, 239-260.

Easterling, D. R., \& Peterson, T. C. (1995). A new method for detecting undocumented discontinuities in climatological time series. International Journal of Climatology, 15, 369-377. 
Hansen, J., \& Lebedeff, S. (1987). Global trends of measured surface air temperature. Journal of Geophysical Research, 92, 13345-13372.

Hansen, J., \& Lebedeff, S. (1988). Global surface air temperatures: Update through 1987. Geophysical Research Letters, 15, 323-326.

Hastie, T., Tibshirani, R., Friedman, J. (2009). Hierarchical clustering. In The elements of statistical learning (2nd Ed.). New York: Springer Verlag.

Honaker, J., King, G., Blackwell, M. (2009). AMELIA II: A Program for Missing Data. Recuperado de http://citeseerx. ist.psu.edu/viewdoc/download?doi=10.1.1.168.2012\&rep= rep1\&type $=$ pdf.

Izzo, M. (2011). Analisi del clima e delle dinamiche climatiche nella Repubblica Dominicana e delle relative influenze sul territorio. (Tesis doctoral). Università degli Studi del Molise, Italia.

Jones, P. D., Wigley, T. M. L., Wright, P. B. (1986a). Global temperature variations between 1861 and 1984. Nature, 322, 430-434.

Jones, P. D., Raper, S. C., Bradley, R. S., Díaz, H. F., Kelly, P. M., Wigley, T. M. L. (1986b). Northern Hemisphere surface air temperature variations, 1851-1984. Journal of Climate and Applied Meteorology, 25, 161-179.

Jones, P. D., Raper, S. C. B., Wigley, T. M. L. (1986c). Southern Hemisphere surface temperature variations: 1851-1984. Joumal of Climate and Applied Meteorology, 25, 1213-1230.

Jones, P. D. (1988). Hemispheric surface air temperature variations: Recent trends and an update to 1987. Journal of Climate, 1, 654-600. 
Análisis de tendencias climáticas basado en metadatos de estaciones de la red de medición de la Oficina Nacional de Meteorología de la República Dominicana

Jones, P. D., \& Briffa, K. R. (1992). Global surface air temperature variations during the twentieth century: Part 1 , spatial, temporal and seasonal details. The Holocene, 2, 174-188.

Jones, P. D., \& Briffa, K. R. (1996). Decadal-to-century timescale variability of regional and hemispheric-scale temperature. En The natural variability of the climate system on 10-100 year timescales. En D. Martinson, K. Bryan, M. Ghil, M. Hall, T. R. Karl, E. S. Sarachik, S. Sorooshian, L. D. Talley. (Eds.). Washington D.C.: Academic Press.

Kelly, P. M., \& Wigley, T. M. L. (1992). Solar cycle length, greenhouse forcing and global climate. Nature, 360, 328-330.

Labeyrie, L., Skinner, L., Cortijo, E. (2007). Sub-Milankovitch (DO/Heinrich) events. In Paleoclimate reconstruction (s.n.), 1964-1974.

Mann, M. E., Bradley, R. S., Hughes, M. K. (1998). Global-scale temperature patterns and climate forcing over the past six centuries. Nature, 392, 779-787.

Mann, M. E., \& Jones, P. D. (2003). 2,000 year hemispheric multi-proxy temperature reconstructions. En IGBP PAGES/ World Data Center for Paleoclimatology Data Contribution Series, 51. [NOAA/NGDC Paleoclimatology Program, Boulder].

Maugeri, M., Brunetti, M., Buffoni, L., Lentini, G., Mangianti, F., Monti, F. (2006). Esame critico e omogeneizzazione delle serie storiche secolari italiane di dati meteorologici e analisi delle tendenze nei dati barometrici. En G. C. Cortemiglia. (Ed.). La variabilità del clima locale relazionata ai fenomeni di cambiamento climatico globale (11-80). Bologna: Patron.

Naciones Unidas. (1992). United Nations Framework Convention on Climate Change. Rio de Janeiro: Naciones Unidas. 
Nanni, T. (2002). Metodi per la valutazione dell'omogeneità e per l'estrazione di segnali da serie storiche di dati meteorologici. Recuperado de http://climagri.entecra.it/9D2C5095-06B34716-9DC0-B425E8EFAEE9/FinalDownload/Download Id-ACE800BB24FE0350E07200759396ACDA/9D2C50 95-06B3-4716-9DC0-B425E8EFAEE9/relazioniFinali/ 2anno/Maugeri_relazione2anno.pdf

Parish, E., Ganguly, A. R., Steinhaeuser, K. (2008). Climate change in the Dominican Republic. (Technical Manual). Tennessee: Oak Ridge National Laboratory.

Parker, D. E., Jones, P. D., Folland, C. K., Bevan, A. (1994). Interdecadal changes of surface temperature since the late 19th century. Journal of Geophysical Research, 99(D7), 1437314399.

Reeves, J., Chen, J., Wang, X. L., Lund, R., Lu, Q. (2007). A review and comparison of changepoint detection techniques for climate data. Journal of Applied Meteorology and Climatology, 46, 900-915.

Rosenzweig, C., Karoly, D., Vicarelli, M., Neofotis, P., Wu, Q., Casassa, G. (2008). Attributing physical and biological impacts to anthropogenic climate change. Nature, 453, 353-357.

Royal Society. (2010). Climate change: a summary of the science. London: The Royal Society.

Rubin, D. B. (1976). Inference and missing data. Biometrika, 63, 581-590.

Rubin, D. B. (1987). Multiple imputation for nonresponse in surveys. New York: John Wiley \& Sons.

Solomon, S., Qin, D., Manning, M., Chen, Z., Marquis, M., Averyt, K. B., Tignor, M., \& Miller, H. L. (Eds.). (2007). Climate Change 2007: The Physical Science Basis. Contribution of Working Group 
Análisis de tendencias climáticas basado en metadatos de estaciones de la red de medición de la Oficina Nacional de Meteorología de la República Dominicana

I to the Fourth Assessment Report of the Intergovernmental Panel on Climate Change. Cambridge: Cambridge University Press.

Wang, X. L. (2003). Comments on detection of undocumented change points: A revision of the two-phase regression model. Journal of Climate, 16, 3383-3385.

Wang, X. L., Wen, Q. H., Wu, Y. (2007). Penalized maximal t test for detecting undocumented mean change in climate data series. Journal of Applied Meteorology and Climatology, 46(6), 916-931.

Wang, X. L. (2008a). Accounting for autocorrelation in detecting mean-shifts in climate data series using the penalized maximal t or F test. Journal of Applied Meteorology and Climatology, 47, 2423-2444.

Wang, X. L. (2008b). Penalized maximal F-test for detecting undocumented mean-shifts without trend-change. Journal of Atmospheric and Oceanic Technology, 25(3), 368-384.

Wang, X. L. (2009). A quantile matching adjustment algorithm for Gaussian data series, Climate Research Division. (Atmospheric Science and Technology Directorate, Science and Technology Branch, Environment Canada). Recuperado de http://cccma.seos.uvic.ca/ETCCDMI/software.shtml.

Wang, X. L., Chen, H., Wu, Y., Pu, Q. (2010). New techniques for detection and adjustment of shifts in daily precipitation data series. Recuperado de http://cccma.seos.uvic.ca/ ETCCDMI/RHtest/transformTPRs.pdf

Wijngaard, J. B., Klein Tank, A. M. G., Können, G. P. (2003). Homogeneity of 20th century european daily temperature and precipitation series. International Journal of Climatology, 23, 679-692.

Zveryaev, I. I., \& Chu, P. S. (2003). Recent climate changes in precipitable water in the global tropics as revealed in National Centers for Environmental Prediction/National Center for 
Atmospheric Research reanalysis. Journal of Geophysical Research, 108, 4311-4320.

\section{Michela Izzo}

Es licenciada en Ciencias Ambientales (Universidad de Molise, Italia). Tiene una maestría en Ingeniería del Viento (Politécnico de Milán, Italia) y un doctorado en Ambiente y Territorio (Universidad de Molise, Italia). Actualmente se desempeña como encargada de Energía Renovable en el Programa de Pequeños Subsidios (PPS-SGP/FMAM) y directora Ejecutiva de Guakia Ambiente, a través del cual apoya las iniciativas de desarrollo local basadas en el empoderamiento de los grupos comunitarios. Es consultora ambiental para la realización de estudios en tema de vulnerabilidad territorial frente al cambio climático. Cuenta con más de diez publicaciones en revistas científicas internacionales sobre temas relacionados con clima y vulnerabilidad territorial.

E-mail: michela.izzo@gmail.com

\section{María Ozoria Zarzuela}

Es ingeniera en sistema de computación egresada de la Universidad Dominicana O \& M. Tiene formación en el sistema de gestión de base de datos sobre el clima en la prevención de desastres naturales y mitigación de los efectos, Snet, San Salvador. Tiene una maestría en telecomunicaciones por la Universidad Autónoma de Santo Domingo (UASD); 
Análisis de tendencias climáticas basado en metadatos de estaciones de la red de medición de la Oficina Nacional de Meteorología de la República Dominicana

un diplomado de cambio climático y adaptación por el Instituto Tecnológico de Santo Domingo.

Es técnica en medio OMM III (meteoróloga clase III) y experta en retos de la hidrometeorología ante las posibles variaciones del clima y los eventos extremos.

E-mail: onamet_zm@yahoo.com

Recibido: 19/09/2013Ａprobado: 10/02/2014 
